\title{
THE NUMBER OF NONISOMORPHIC BOOLEAN SUBALGEBRAS OF A POWER SET
}

\author{
FRANCISCO J. FRENICHE ${ }^{1}$
}

\begin{abstract}
It is shown that if $\kappa$ is an infinite cardinal, then there are $2^{2^{\kappa}}$ nonisomorphic Boolean subalgebras of $P(\kappa)$. Also it is shown that if $\kappa=c$, then the above subalgebras can be choosen countably complete. This solves a question raised by S. Ulam.
\end{abstract}

In $[3$, p. 106] the following questions are raised by Ulam:

(a) How many nonisomorphic Boolean algebras of sets of integers exist?

(b) How many nonisomorphic countably complete Boolean algebras of sets of real numbers exist?

The answer to the first question is almost trivial, as was independently noted by E. K. van Douwen (partial results were obtained independently by S. Williams).

We refer the reader to $[\mathbf{2}]$ for unexplained notions.

Let $\kappa$ be an infinite cardinal. A subalgebra $A$ of $P(\kappa)$ is full if $\{\alpha\} \in A$ for every $\alpha \in \kappa$.

LEMMA 1. If $\lambda$ is the number of full subalgebras of $\mathcal{P}(\kappa)$, and $\lambda>2^{\kappa}$, then $P(\kappa)$ has $\lambda$ nonisomorphic full subalgebras.

PROOF. Any isomorphism between full subalgebras of $P(\kappa)$ is induced by a permutation of $\kappa$. Hence each isomorphism class of full subalgebras has at most $2^{\kappa}$ elements. Thus there are $\lambda$ classes, as desired.

This lemma and proof are essentially found in [4].

If $F$ is a filter on a Boolean algebra $A$, let $-F=\{-a: a \in F\}$. It is well known, and easy to see, that $F \cup-F$ is a subalgebra of $A$, which we denote by $A_{F}$.

LEMMA 2. If $F$ and $G$ are distinct filters on $A$, not ultrafilters, then $A_{F} \neq A_{G}$.

Proof. Say $b \in F \backslash G$. If also $-b \notin G$, then $b \in A_{F} \backslash A_{G}$. Suppose $-b \in G$. Choose $c \in A$ so that $c,-c \notin F$. Then $-c \vee-b \in G$ while clearly $c \wedge b,-c \vee-b \notin F$. Thus $c \wedge b \in A_{G} \backslash A_{F}$.

THEOREM 1. $P(\kappa)$ has $2^{2^{\kappa}}$ nonisomorphic full subalgebras.

PROOF. By Lemma 1 it suffices to exhibit $2^{2^{\kappa}}$ full subalgebras. Let $A$ consist of all filters which are the intersection of two free ultrafilters. Clearly $P(\kappa)_{F}$ is full for each $F \in A$. Now if $F_{i}$ is a free ultrafilter for $i=1,2,3,4$, and $F_{1} \neq F_{2} \neq F_{3} \neq F_{4}$,

Received by the editors May 9, 1983 and, in revised form, September 7, 1983.

1980 Mathematics Subject Classification. Primary $06 \mathrm{E} 05$.

Key words and phrases. Boolean algebra, almost disjoint family, large oscillation family.

${ }^{1}$ This is a slightly modified part of the author's $\mathrm{Ph}$. D. Thesis prepared at the Universidad de Sevilla under the supervision of Professor Juan Arias de Reyna. I wish to thank Professor Arias de Reyna for his interest and advice.

(C) 1984 American Mathematical Society $0002-9939 / 84 \$ 1.00+\$ .25$ per page 
$F_{1} \neq F_{3}$, then $F_{1} \cap F_{2} \neq F_{3} \cap F_{4}$. For, say $a \in F_{1} \backslash F_{3}, b \in F_{2} \backslash F_{3}$. Then $a \cup b \in$ $\left(F_{1} \cap F_{2}\right) \backslash\left(F_{3} \cap F_{4}\right)$, as desired. Since there are $2^{2^{\kappa}}$ free ultrafilters by Pospisil's theorem [2, p. 146], the theorem follows by Lemma 2 .

The above ideas can be used to treat the second question also. Let us call a filter $F$ on $P(\kappa)$ full if $\kappa \backslash\{\alpha\} \in F$ for all $\alpha \in \kappa$ and $F$ is not an ultrafilter; thus $P(\kappa)_{F}$ is then a full subalgebra of $P(\kappa)$. If $\lambda$ is a cardinal and $F$ is $\lambda$-complete, then so is $P(\kappa)_{F}$. We are interested in counting the number of $\lambda$-complete full filters on $P(\kappa)$.

We extend here some results of A. Tarski [5].

THEOREM 2. Suppose there is a family $\mathcal{F}$ of subsets of $\kappa$, which is $\kappa$-almost disjoint. Let $|\mathcal{F}|=\lambda \geq \omega$. Then $\mathcal{P}(\kappa)$ has at least $2^{\lambda} \operatorname{cf}(\kappa)$-complete full filters.

Proof. For each subset $H$ of $₹$ let

$$
F_{H}=\{a \subset \kappa:|b \backslash a|<\kappa \text { for all } b \in H\} .
$$

Clearly $F_{H}$ is a $\operatorname{cf}(\kappa)$-complete filter on $\kappa$ and $\kappa \backslash\{\alpha\} \in F_{H}$ for every $\alpha \in \kappa$.

If $|H|>1$, then $F_{H}$ is not an ultrafilter. For, choose distinct $a, b \in H$. Then $\kappa \backslash a \notin F_{H}$. Since $|a \cap b|<\kappa$, we have $|b \backslash a|=\kappa$, and so $a \notin F_{H}$. Thus, indeed, $F_{H}$ is not an ultrafilter. So $F_{H}$ is a full filter.

If $H, K \in \mathcal{F}$ and $H \neq K$, say $a \in H \backslash K$, then $\kappa \backslash a \in F_{K} \backslash F_{H}$. Hence the theorem follows.

For exhaustive information on when the hypothesis of Theorem 2 can happen, see [1]. In particular (a result of W. Sierpiński), there is always such a family $\mathcal{F}$ of size $\kappa^{+}$, so by the earlier results we have

COROLlARY. If $2^{\kappa}<2^{\kappa^{+}}$, then there are at least $2^{\kappa^{+}}$nonisomorphic $\operatorname{cf}(\kappa)$ complete full subalgebras of $P(\kappa)$.

THEOREM 3. If $\lambda$ is regular and $\kappa^{\lambda}=\kappa$, then there are $2^{2^{\kappa}} \lambda$-complete full filters on $P(\kappa)$, and hence $2^{2^{\kappa}}$ nonisomorphic $\lambda$-complete full subalgebras of $P(\kappa)$.

ProOF. Since $\omega \leq \kappa \leq \kappa^{\lambda}=\kappa$, we can choose a $\lambda$-large oscillation family $S \cup\{d\}$ of subsets of $\kappa$, with $|S|=2^{\kappa}$ and $d \notin S$ [2, Corollary 3.17].

For each subset $H$ of $S$ let $F_{H}=\left\{a \subset \kappa\right.$ : there exist $\Gamma \in[H]^{<\lambda}$ and $\Delta \in$ $[S \backslash H]^{<\lambda}$ such that $\left.(\bigcap\{b: b \in \Gamma\}) \cap(\bigcap\{\kappa \backslash c: c \in \Delta\}) \subset a\right\}$. Clearly $F_{H}$ is a $\lambda$ complete filter on $\kappa$.

We claim that there exists a subset $C$ of $P(S)$ with $|C|=2^{2^{\kappa}}$ such that $\kappa \backslash\{\alpha\} \in$ $F_{H}$ for every $\alpha \in \kappa$ and $H \in C$. Indeed, since $2^{\kappa}<\operatorname{cf}\left(2^{2^{\kappa}}\right)$, there exist a subset $C$ of $P(S)$ with $|C|=2^{2^{\kappa}}$ and a subset $a$ of $\kappa$ such that $a=\bigcap\left\{b: b \in F_{H}\right\}$ for all $H \in C$. We have $a=\emptyset$. For, choose distinct $H, K \in C$, say $b \in H \backslash K$. Then $b \in F_{H}, \kappa \backslash b \in F_{K}$ and so $a \subset b \cap(\kappa \backslash b)$. It is clear that $C$ is as desired.

Finally, since $d, \kappa \backslash d \notin F_{H}, F_{H}$ is not an ultrafilter. Thus $F_{H}$ is a $\lambda$-complete full filter on $\kappa$ and the theorem follows.

COROLLARY. There are $2^{2^{c}}$ nonisomorphic countably complete full subalgebras of $P(\mathbf{R})$.

PROOF. $c^{\omega 1}=c^{\omega}=c$, so this follows from the theorem.

ACKNOWLEDGEMENTS. I am indebted to the referee for this valuable suggestion. 


\section{REFERENCES}

1. J. Baumgartner, Almost-disjoint sets, the dense set problem and the partition calculus, Ann. Math. Logic 9 (1976), 401-439.

2. W. W. Comfort and S. Negrepontis, The theory of ultrafilters, Springer-Verlag, Berlin, 1974.

3. R. D. Mauldin (editor), The Scottish book, Birkhäuser, Boston, Mass., 1981.

4. W. Rudin, Homogeneity properties in the theory of Cech compactifications, Duke Math. J. 23 (1956), 409-419.

5. A. Tarski, Ideals in vollständigen Mengenkörpern. I, Fund. Math. 32 (1939), 45-63.

Facultad de Matemáticas, Universidad de Sevilla, C/Tarfia SN, Sevilla-12, SPAIN 